\title{
Assessing the Meaning of Emojis for Emotional Awareness - A Pilot Study
}

\author{
Wesley Brants \\ Eindhoven University of Technology \\ Eindhoven, The Netherlands \\ w.m.g.brants@student.tue.nl
}

\author{
Bonita Sharif \\ University of Nebraska - Lincoln \\ Lincoln, Nebraska, USA \\ bsharif@unl.edu
}

\author{
Alexander Serebrenik \\ Eindhoven University of Technology \\ Eindhoven, The Netherlands \\ a.serebrenik@tue.nl
}

\begin{abstract}
Emojis are increasingly being used in today's social communication - both formally in team messaging systems as well as informally via text messages on phones. Besides being used in social communication, emojis might also be a suitable mechanism for emotion (self-)assessment. Indeed, emojis can be expected to be familiar to people of different social groups and do not depend on the mastery of a specific language. However, emojis could be interpreted very differently from their actual intent. In order to determine whether people interpret emojis (specific to emotional states) in a consistent manner, we conducted an online survey on nine emojis with 386 people. The results show that the emojis representing anger, sadness, joy, surprise, and neutral state are interpreted as they were intended, independent of age and gender. Interpretations of other emojis such as Unamused Face and Face Screaming in Fear depend on age, and thus are not as useful for probing for emotion in a study setting unless all participants belong to the same age category. The Face with Rolling Eyes emoji is interpreted differently by gender and finally the Nauseated Face emoji resulted in no conclusive interpretation.
\end{abstract}

\section{CCS CONCEPTS}

- Human-centered computing $\rightarrow$ Empirical studies in HCI; • Social and professional topics $\rightarrow$ Gender; Age.

\section{KEYWORDS}

emoji interpretation; pilot study; emotional awareness, gender, age

ACM Reference Format:

Wesley Brants, Bonita Sharif, and Alexander Serebrenik. 2019. Assessing the Meaning of Emojis for Emotional Awareness - A Pilot Study. In Companion Proceedings of the 2019 World Wide Web Conference (WWW'19 Companion), May 13-17, 2019, San Francisco, CA, USA. ACM, New York, NY, USA, 5 pages. https://doi.org/10.1145/3308560.3316550

\section{INTRODUCTION}

Emotions and their awareness can have a big impact on the success or failure of a project, both from a manager's [3] and employee's [7] perspective. This holds for collaborative projects in general, including software development projects [28]. The overall goal of our research is to provide members of collaborative teams with ways of indicating their emotion to other team members; however, to reduce

This paper is published under the Creative Commons Attribution 4.0 International (CC BY 4.0) license. Authors reserve their rights to disseminate the work on their personal and corporate Web sites with the appropriate attribution.

WWW'19 Companion, May 13-17, 2019, San Francisco, CA, USA

(C) 2019 IW3C2 (International World Wide Web Conference Committee), published under Creative Commons CC-BY 4.0 License.

ACM ISBN 978-1-4503-6675-5/19/05.

https://doi.org/10.1145/3308560.3316550 the negative impact of interruption, soliciting emotion reporting should be accurate and quick. Both emojis and words can be used to convey the current mood of a subject, however emojis (and images in general) are more easy to interpret and recall than written words. This effect is known as the "picture superiority effect" and is due to dual-encoding of the pictures in the brain as an image and as a verbal trace [5, 22]. Moreover, use of graphic representations also works better for people who are dyslexic or otherwise aphasic. Lastly, there is no language barrier to consider.

Emojis are popular and used frequently used in many different settings [17]. However, due to the different representation of emojis on each platform and device [18], there could be a difference in perception of sentiment conveyed by an emoji. Moreover, sentiment might be misconstrued due to the same visual representation being interpreted differently [18]. Thus using emojis as indicator of one's emotional state might result in false conclusions if a person interprets an emoji differently as was intended by the researcher.

In this work, we analyze differences in interpretation of emotionrelated emojis. Specifically, we consider the impact of gender and age on the interpretation of emotion-related emojis as gender and age have been shown to affect the use of emojis [21,27]. We do this as part of assessing the suitability of emojis in probing for emotional awareness state.

Our results summarized in Table 1 show that interpretation of two of the nine emotion-related emojis are age-dependent, and one is gender-dependent. This means that using these three emojis to probe the emotional state of subjects should only be done in presence of gender- or age-homogeneous groups.

\section{RELATED WORK}

Measuring the emotional state of subjects can be done via several techniques. One category of techniques observes the subject, an activity or analyzes an artifact to infer the emotion of the participant. An example of this category of techniques is a lexicon approach, in which the emotional state is deducted from the analysis of words and sentences in which emotional words (i.e. love, shame, etc.) are given an emotional weight. There are multiple tools [13] that can be used for this such as SentiStrength [25], Stanford NLP sentiment analyser [24] and NLTK [16]. Moreover, importance of domainspecific aspects has been recognized, e.g., for software engineering [14] triggering development of a series of sentiment analysis tools targeting this particular domain. Another method of inferring the emotional state of a subject is the use biometric data such as pupil size, heart rate $[8,20]$ and galvanic skin response.

A second category of techniques to capture the emotional state is to explicitly ask the subject to indicate their his or her emotional state. This can for example be done prior to the task at hand [12]. There are 
Table 1: Is interpretation of emojis independent from gender and age?

\begin{tabular}{lrcc}
\hline Description & Emoji Gender Age \\
\hline Pouting Face & $\ddots 6$ & $\checkmark$ & $\checkmark$ \\
Unamused Face & $\ddots$ & $\checkmark$ & $x$ \\
Nauseated Face & $\ddots$ & $\checkmark$ & $\checkmark$ \\
Face Screaming in Fear & 600 & $\checkmark$ & $x$ \\
Face with Rolling Eyes & -0 & $x$ & $\checkmark$ \\
Crying Face & 0 & $\checkmark$ & $\checkmark$ \\
Face with Tears of Joy & $\ddots$ & $\checkmark$ & $\checkmark$ \\
Face with Open Mouth & $\ddots$ & $\checkmark$ & $\checkmark$ \\
Neutral Face & $\ddots$ & $\checkmark$ & $\checkmark$ \\
\hline
\end{tabular}

several ways to do so; one can use a scale to indicate to what extent the participant is feeling an emotion, or one can do this via a standardized questionnaire such as Profile of Mood States (POMS) [15] or Positive and Negative Affect Schedule (PANAS) [26]. Other techniques besides these interviews and questionnaires are the use of film and pictures (e.g., the International Affective Picture System (IAPS)) [4]. Due to the picture superiority effect, using visual cues is not a strange choice. Combined with the familiarity of emojis and all benefits over written words it gives as explained before, emojis seem a logical choice for probing emotional state of the participant.

We report on studies done on the use of emoticons and emojis. Previous research shows that females are more accurate than males in judging emotional meaning from nonverbal cues [9]. Hudson et al. report on a study of emoticons showing that females are more jealous on Facebook, but when a winking emoticon ; ) was present, males were more jealous [10]. Also, studies have shown that females have given more emotional dimensions to emoticons than males, meaning they interpret emoticons in more ways than do males [27]. A study on the use of emoticons in Japan hypothesized that females use emoticons more frequently than males. Also, younger people would use emoticons more frequently as compared to older people [21].

Emojis are quite different from emoticons. Emoticons are strings of characters representing some meaning such as sad or happy; emojis are images with little details conveying a more subtle meaning [18]. In the current literature, emojis have not yet been researched to the same extent as emoticons.

It has been shown that males and females use emojis differently and give them different sentiments. Also, there is a difference in frequency of emoji use depending on the time of the day, week and year [2]. For example, the emojis representing Christmas trees are used more often in December. The use of emojis has been shown to be different between males and females where different emojis are used at different times of the day, week and year as well as using different sentiments for emojis. Age might also be a factor that influences usage as different generations communicate in different ways. Nishimura et al. hypothesized that since younger generations
Table 2: Age Distribution of 386 respondents

\begin{tabular}{lr|lr}
\hline Age & Participants & Age & Participants \\
\hline $18-23$ & 67 & $36-41$ & 55 \\
$24-29$ & 110 & $\geq 42$ & 79 \\
$30-35$ & 72 & did not answer & 3 \\
\hline
\end{tabular}

have more experience with emojis, they would use them more frequently [21]. This hypothesis was not supported by the results.

Research on the usage of emojis in food quality surveys has shown that emoji interpretation and frequency of use was not influenced by gender. However, when emojis are used to measure productrelated emotional associations, age-related differences exist [11]. Our research has a different goal of determining whether emojis are suitable to probe emotional awareness, rather than rating a product.

\section{STUDY OVERVIEW}

The goal of this study is to infer whether emotion-related emojis are indeed a suitable way of determining the emotional state of a participant in a research study. This can only be done if the interpretations of emojis do not vary too much. To generalize the application of the results of this work, we survey a diverse group of participants. Since the usage of emojis is influenced by gender and age we ask the following research question Does gender or age influence the interpretation of an emoji? Based on the answer obtained we can also determine what emojis are suited for determining emotional awareness in gender and age diverse target groups.

We use nine emotion-related emojis for this study as shown in Table 1. Emojis are presented according to the Unicode style used by Apple Inc. These emojis were chosen because they are most commonly associated with emotions. Seeing whether interpretations are different in different age and gender categories is not entirely new. For some emojis the interpretation and usage of these emojis in questionnaires have been researched [11]. However, we perform this research with a different goal in mind; namely if they are usable in research in which emotional state is important. Moreover, we partly use other emojis which seem fit for this kind of goal. In order to answer the research question, we created an online survey and advertised it on Facebook and user groups. The participants were first asked to indicate their gender and age. Then, they were presented with 9 emojis in Table 1 and were asked to answer the question "What emotion is this emoji displaying?". The participants were allowed to provide a free-form answer in any language, and were not restricted to any length in response. We did this to obtain information that is as rich as possible [6]. A total of 389 people participated of which 3 responses were discarded.

\section{DATA LABELING}

The survey resulted in many different kinds of responses, varying from single words ("Bored", "Anger") to descriptive sentences ("it's like well I prefer not to comment about it"). We did not remove spelling mistakes or alter the responses in any way before labeling. We identify four response categories.

(1) One word in English, representing an emotion

(2) One word in English, not representing an emotion 
(3) Multiple words or descriptive responses in English

(4) Non-English responses

A response in the first category was chosen as specified in Plutchik's wheel of emotions [23] because this model has eight basic emotions: Anger, Disgust, Sadness, Surprise, Fear, Trust, Joy and Anticipation. For the single-word responses that could not be directly mapped to the emotions from Plutchik's framework, we used WordNet and the website http://www.thesaurus.com to determine synonyms. The second category was a response that was clearly not an emotion such as "alien sneezing".

The third category contained multiple words or sentences, and sometimes one of the words in the response was a basic emotion. In this case, the response was categorized under that basic emotion category. To prevent oversimplification of the interpretation of the responses, we decided not to do the same if the response included a synonym or a related emotion. Also responses such as "angry or disgusted" were not categorized automatically: WordNet lemmatizers consider for example 'anger' and 'angry' to be different words as they are different parts of speech. Responses that could not be automatically mapped to emotions, were manually labeled by each of the authors taking an average of 5 hours per labeler. A detailed labeling manual created by the first author was used for the labeling task. The labelers were allowed to choose from 11 options; the eight basic emotions from Plutchik's wheel, "Lack of Awareness", "Lack of Emotion", and "Not Possible". The labelers were explicitly instructed, in the case multiple emotions were relevant, to choose the most relevant emotion, and only to resort to "Not Possible" as the last option. Due to moderate agreement, we chose to use a majority vote to set a label. The responses labeled differently by each labeler were discussed during a joint meeting that lasted 3 hours. The fourth category consisted of all non-English responses. All responses were labeled by native speakers of that language using the same detailed labeling manual used in the third category.

After labeling and categorization, we created two contingency tables for each emoji; one with labels and gender and the other with labels and age categories. Because we did not have enough data regarding participants who did not identify as male or female, we chose to exclude these responses. Some emojis had a clear and consistent interpretation, for these emojis no statistical analysis was performed. For the other emojis, we perform a chi-square $\left(\chi^{2}\right)$ test. The null hypothesis of $\chi^{2}$ states that the interpretation of the emoji is independent on the category (gender or age, in our case). The alternative hypothesis states the opposite, i.e., the interpretation depends on the category. For some emojis, not all cells in the contingency table have a value larger than 5 , we chose to combine the rows in which at least one of the cells is lower than 5. This is necessary to make the chi-square test work $[1,19]$.

\section{STUDY RESULTS}

Responses were given in English (3342), Dutch (57), Russian (50), French (8) and Portuguese (17). In total, we had 3474 responses for all emojis, of which 1380 were unique. After initial labeling, Kappa's agreement between the three labelers was moderate (0.468). After discussing the 180 responses for which no majority vote could be applied, an agreement was reached on all of them. The age distribution can be found in Table 2. From the 386 participants, 224 were male, 148 were female, 11 non binary and 3 responded as "Other".

We start by observing that across all gender and age categories, at least $94 \%$ of the respondents recognize the Pouting Face emoji as anger. This is by far the most agreed upon emoji in our collection. Similarly, clear agreement across all gender and age categories has been observed for such emojis as Face with Tears of Joy $\Theta$ (joy), Face with Open Mouth $\because$ (surprise) and Crying Face $\left(0^{\circ}\right.$ (sadness). In Table 7 the results can be seen for each emoji per category. The text under the emoji is the most common interpretation of the emoji. Each cell gives the amount of responses that gave this response. The numbers under total represent the number of participants in that category, and are put there for easy reference.

Next, we observed that the suitability of several emojis to probe for emotional state in gender- and age-diverse target groups vary per emoji. The interpretation of the Unamused Face emoji

$\Theta$ differs per age category: younger people are more inclined to interpret this emoji as anger, whereas this shifts to disgust and further towards other interpretations (of which the biggest share comes from sadness) as age progresses (see also Table 4). This observation that the interpretation of the Unamused Face emoji depends on age is further supported by the $\chi^{2}$ test $(p<0.0001)$. However, with respect to gender, we cannot reject the null hypothesis $\left(\chi^{2}=7.423, d=\right.$ $5, p=0.191)$, hence we cannot say the interpretation of this emoji is influenced by gender. This observation concurs and reinforces the earlier observation of Miller et al. [18] that the Unamused Face emoji has the highest semantic misconstrual score among the Apple emojis studied.

Similarly, in case of the Face Screaming in Fear emoji bed, the interpretation shifts from surprise to fear from younger to older age groups, see Table 6. This indicates that the Unamused Face and Face Screaming in Fear emoji can only be used if all participants are in the same age category, but results might be incorrect if young and older people are mixed within the study. Hence, one needs to be cautious when using Unamused Face and Face Screaming in Fear emojis for emotional awareness.

The Face With Rolling Eyes emoji $\bigcirc$ is age independent, but gender dependent; here females indicate the emoji as anger, whereas males interpret it more neutrally. The results for this emoji can be seen in Table 3. Hence using this emoji in a study containing both genders might give incorrect results as well. Since there is a clear emoji for anger (See Figure 1), we would advise not to use the rolling eyes emoji, especially not in gender-diverse groups.

Finally, the Nauseated Face $\odot$ and Neutral Face $\odot$ emojis are interpreted independently by gender and age. For the Nauseated Face emoji, although there is a small favor towards digust, there is no clear winner in all cases. Quite often it is seen as an object not representing emotion but, e.g., physical condition such as vomiting. When analyzing the contingency tables, for the Nauseated Face emoji, there is a majority representing disgust, which is in accordance with one of the definitions from Emojipedia. However, there is a close runner up, namely Lack of Emotion. See Table 5 for a clustered contingency table for the Nauseated Face emoji. This is in 
Table 3: Clustered contingency table for gender and the Face With Rolling Eyes emoji. The Other category stands for Fear, Trust, Joy and Anticipation

\begin{tabular}{|c|c|c|c|c|c|c|c|c|c|}
\hline & Anger & Disgust & Sadness & Surprise & Lack of Awareness & Lack of Emotion & Not Possible & Other & Total \\
\hline Male & 47 & 24 & 9 & 24 & 17 & 65 & 25 & 13 & 224 \\
\hline Female & 53 & 19 & 5 & 13 & 10 & 32 & 7 & 9 & 148 \\
\hline Total & 100 & 43 & 14 & 37 & 27 & 97 & 32 & 22 & 372 \\
\hline
\end{tabular}

Table 4: Clustered contingency table for age and the Unamused face emoji. The Other category stands for Sadness, Surprise, Fear, Trust, Joy, Anticipation and Lack of Awareness

\begin{tabular}{c|cccc|c}
\hline Age Range & Anger & Disgust & $\begin{array}{c}\text { Lack of } \\
\text { Emotion }\end{array}$ & Other & Total \\
\hline $18-23$ & 36 & 13 & 8 & 10 & 67 \\
$24-29$ & 24 & 23 & 27 & 36 & 110 \\
$30-35$ & 14 & 22 & 12 & 24 & 72 \\
$36-41$ & 11 & 18 & 6 & 20 & 55 \\
$42+$ & 8 & 17 & 9 & 45 & 79 \\
\hline Total & 93 & 93 & 62 & 135 & 383 \\
\hline
\end{tabular}

Table 5: Clustered contingency table for gender and the Nauseated Face emoji. The Other category stands for Anger, Sadness, Surprise, Trust, Joy, Anticipation and Lack of Awareness.

\begin{tabular}{cccccc|c}
\hline Gender & Disgust & Fear & $\begin{array}{c}\text { Lack of } \\
\text { Emotion }\end{array}$ & $\begin{array}{c}\text { Not } \\
\text { Possible }\end{array}$ & Other & Total \\
\hline Male & 112 & 5 & 74 & 15 & 18 & 224 \\
Female & 81 & 5 & 45 & 6 & 11 & 148 \\
\hline Total & $\mathbf{1 9 3}$ & $\mathbf{1 0}$ & $\mathbf{1 1 9}$ & $\mathbf{2 1}$ & $\mathbf{2 9}$ & $\mathbf{3 7 2}$ \\
\hline
\end{tabular}

Table 6: Clustered contingency table for age and the Face Screaming in Fear emoji.

\begin{tabular}{c|cc|c}
\hline Age Range & Surprise & Fear & Total \\
\hline $18-23$ & 43 & 19 & 62 \\
$24-29$ & 61 & 41 & 102 \\
$30-35$ & 34 & 31 & 65 \\
$36-41$ & 21 & 31 & 52 \\
$42+$ & 30 & 37 & 67 \\
\hline Total & $\mathbf{1 8 9}$ & $\mathbf{1 5 9}$ & $\mathbf{3 4 8}$ \\
\hline
\end{tabular}

contradiction with its definition on Emojipedia. It would thus not be useful to include this emoji when probing for one's emotional state.

The Neutral Face emoji should represent a neutral state, and although the emoji is also often interpreted as surprise (especially by males), lack of emotion is the most often used interpretation. The Neutral Face emoji can be used for finding an emotional baseline.

Main Finding: Basic emotions of anger, surprise, joy and sadness had conclusive results and were understood as intended by their corresponding emoji. However, disgust and fear (for younger age groups) did not have conclusive results. Younger people are more inclined to interpret the Unamused Face emoji as anger.
Table 7: Most common response per category per emoji

\begin{tabular}{c|cccc|c}
\hline Category & $\begin{array}{c}\Theta \\
\text { (Anger) }\end{array}$ & $\begin{array}{c}\Theta \\
(\text { Joy })\end{array}$ & $\begin{array}{c}\because \\
\text { (Surprise) }\end{array}$ & $\begin{array}{c}(6) \\
\text { (Sadness) }\end{array}$ & Total \\
\hline Male & 221 & 213 & 179 & 214 & 224 \\
Female & 144 & 143 & 127 & 141 & 148 \\
$18-23$ & 66 & 66 & 61 & 65 & 67 \\
$24-29$ & 109 & 104 & 97 & 105 & 110 \\
$30-35$ & 72 & 69 & 66 & 68 & 72 \\
$36-41$ & 54 & 53 & 48 & 53 & 55 \\
$42+$ & 75 & 74 & 63 & 75 & 79 \\
\hline
\end{tabular}

Table 8: Summary of the emojis' interpretation

\begin{tabular}{c|c}
\hline Emoji & Emotional State Interpretation \\
\hline$\because 6$ & Anger \\
\hline$\because 0$ & Anger, Disgust, Sadness, Lack of Emotion \\
\hline$\because$ & Disgust, Lack of Emotion \\
\hline$\because 0$ & Fear, Surprise \\
\hline 00 & Anger, Disgust, Surprise, Lack of Emotion \\
\hline$\because 0^{\circ}$ & Sadness \\
\hline$\because$ & Joy \\
\hline$\because$ & Surprise \\
\hline$\because$ & Lack of Emotion \\
\hline
\end{tabular}

\section{THREATS TO VALIDITY}

It is possible that people's use of emojis is highly dependent on the context it is used in. However our goal was to situate this in a more general context of emotion awareness and to do this, only emojis related to basic emotions were used. It is also possible that other factors besides age and gender such as culture, disability, socioeconomic status, occupation and the context in which the emoji is used (to name a few) could affect the results. However, we wanted to start by looking at age and gender in particular and later expand to include other factors in a larger followup study.

\section{CONCLUSIONS AND FUTURE WORK}

The paper presents the results of an online survey seeking to determine the validity of using emojis for emotional awareness of the 
participant in a research study. The results show that six basic emotions of anger, disgust, fear, surprise, joy and sadness had conclusive results and were understood as intended. However, disgust and fear (for younger people) did not have conclusive results. To answer our research question, we can thus conclude that there are emojis (Unamused Face, Nauseated Face and Face With Rolling Eyes) that are being interpreted differently depending on age or gender. However, there is a collection of emojis (Pouting Face, Crying Face, Face with Tears of Joy, Face with Open Mouth, Neutral Face) which are indeed suited for determining emotional awareness in gender and age diverse target groups. Our goal was to situate in a general context of emotion awareness. As we needed to start somewhere, we only considered gender and age. However, other factors such as context and demographic and cultural factors also play a role in the interpretation of an emoji. These threats to validity should be considered in future work. Other setups for research can be thought if as well. For example, the participant must choose between several emojis, or must label text sentimentally with emojis.

As part of our future work, we are interested in learning how software developers use emojis, and to see whether it can then be inferred from emoji use what the emotional state of the software developer is. Emojis can also help improve the working environment, improve user friendliness and help the developer to become emotionally aware of his/her own emotions. Besides more research needed on culture and other emotional models with other labelers, as some persons who did not identify as male or female participated in the survey. These results might be different. Besides this, further research can include the interpretations of other emojis to see whether different ones or different visualizations of emojis obtain better results. This research presents a systematic analysis which will be useful to research similar kinds of questions on this topic.

\section{ACKNOWLEDGMENTS}

This work is supported in part by grants from the National Science Foundation under grant numbers CCF 18-55756 and CCF 15-53573. We are grateful to Alexandra Fountaine, who provided support and ideas for the survey. We thank all volunteers who participated in our survey. We are also thankful to Stephan van den Berkmortel, Weslley Silva Torres and Nathan Pottier for labeling non-English responses.

\section{REFERENCES}

[1] Alan Agresti and Christine A Franklin. 2007. Statistics: The Art and Science of Learning from Data (3rd). (2007).

[2] Zhenpeng Chen, Xuan Lu, Sheng Shen, Wei Ai, Xuanzhe Liu, and Qiaozhu Mei. 2017. Through a Gender Lens: An Empirical Study of Emoji Usage over Large-Scale Android Users. arXiv preprint arXiv:1705.05546 (2017)

[3] Nicholas Clarke. 2010. Projects are emotional: How project managers' emotional awareness can influence decisions and behaviours in projects. International Journal of Managing Projects in Business 3, 4 (2010), 604-624.

[4] James A Coan and John JB Allen. 2007. Handbook of emotion elicitation and assessment. Oxford university press.

[5] Pauline Dewan. 2015. Words Versus Pictures: Leveraging the Research on Visual Communication. Partnership: the Canadian Journal of Library and Information
Practice and Research 10, 1 (2015).

[6] William Foddy and William H Foddy. 1994. Constructing questions for interviews and questionnaires: Theory and practice in social research. Cambridge university press.

[7] Octavio García, Jesús Favela, and Roberto Machorro. 1999. Emotional awareness in collaborative systems. In String Processing and Information Retrieval Symposium, 1999 and International Workshop on Groupware. IEEE, 296-303.

[8] Daniela Girardi, Filippo Lanubile, Nicole Novielli, Luigi Quaranta, and Alexander Serebrenik. 2019. Towards Recognizing the Emotions of Developers Using Biometrics: The Design of a Field Study. In International Workshop on Emotion Awareness in Software Engineering.

[9] Judith A Hall and David Matsumoto. 2004. Gender differences in judgments of multiple emotions from facial expressions. Emotion 4, 2 (2004), 201.

[10] Michael B Hudson, Sylis C Nicolas, Molly E Howser, Kristen E Lipsett, Ian W Robinson, Laura J Pope, Abigail F Hobby, and Denise R Friedman. 2015. Examining how gender and emoticons influence Facebook jealousy. Cyberpsychology, Behavior, and Social Networking 18, 2 (2015), 87-92.

[11] Sara R Jaeger, Yixun Xia, Pui-Yee Lee, Denise C Hunter, Michelle K Beresford, and Gastón Ares. 2018. Emoji questionnaires can be used with a range of population segments: Findings relating to age, gender and frequency of emoji/emoticon use. Food Quality and Preference 68 (2018), 397-410.

[12] Eva Jonas, Verena Graupmann, and Dieter Frey. 2006. The influence of mood on the search for supporting versus conflicting information: Dissonance reduction as a means of mood regulation? Personality and Social Psychology Bulletin 32, 1 (2006), 3-15.

[13] Robbert Jongeling, Subhajit Datta, and Alexander Serebrenik. 2015. Choosing your weapons: On sentiment analysis tools for software engineering research. In 2015 IEEE International Conference on Software Maintenance and Evolution (ICSME). IEEE, 531-535.

[14] Robbert Jongeling, Proshanta Sarkar, Subhajit Datta, and Alexander Serebrenik. 2017. On negative results when using sentiment analysis tools for software engineering research. Empirical Software Engineering 22, 5 (2017), 2543-2584.

[15] James Lani. [n. d.]. Profile of Mood States (POMS). ([n. d.]).

[16] Edward Loper and Steven Bird. 2002. NLTK: The natural language toolkit. In Workshop on Effective tools and methodologies for teaching natural language processing and computational linguistics. Association for Computational Linguistics, $63-70$.

[17] Xuan Lu, Wei Ai, Xuanzhe Liu, Qian Li, Ning Wang, Gang Huang, and Qiaozhu Mei. 2016. Learning from the ubiquitous language: an empirical analysis of emoji usage of smartphone users. In Proceedings of the 2016 ACM International Joint Conference on Pervasive and Ubiquitous Computing. ACM, 770-780.

[18] Hannah Miller, Jacob Thebault-Spieker, Shuo Chang, Isaac Johnson, Loren Terveen, and Brent Hecht. 2016. "Blissfully happy" or "ready to fight": Varying interpretations of emoji. AAAI press, 259-268.

[19] Douglas C Montgomery and George C Runger. 2011. Applied statistics and probability for engineers (5th). John Wiley \& Sons.

[20] Sebastian C Müller and Thomas Fritz. 2015. Stuck and frustrated or in flow and happy: sensing developers' emotions and progress. In ICSE. IEEE, 688-699.

[21] Yukiko Nishimura. 2016. A sociolinguistic analysis of emoticon usage in Japanese blogs: Variation by age, gender, and topic. AoIR Selected Papers of Internet Research 5 (2016).

[22] Allan Paivio and Kalman Csapo. 1973. Picture superiority in free recall: Imagery or dual coding? Cognitive psychology 5, 2 (1973), 176-206.

[23] Robert Plutchik. 2001. The nature of emotions: Human emotions have deep evolutionary roots, a fact that may explain their complexity and provide tools for clinical practice. American scientist 89, 4 (2001), 344-350.

[24] Richard Socher, Alex Perelygin, Jean Wu, Jason Chuang, Christopher D Manning, Andrew Ng, and Christopher Potts. 2013. Recursive deep models for semantic compositionality over a sentiment treebank. In EMNLP. 1631-1642.

[25] Mike Thelwall, Kevan Buckley, Georgios Paltoglou, and Di Cai. 2010. Sentiment in short strength detection informal text. JASIST 61, 12 (2010), 2544-2558.

[26] David Watson, Lee Anna Clark, and Auke Tellegen. 1988. Development and validation of brief measures of positive and negative affect: the PANAS scales. Journal of personality and social psychology 54, 6 (1988), 1063.

[27] Alecia Wolf. 2000. Emotional expression online: Gender differences in emoticon use. CyberPsychology \& Behavior 3, 5 (2000), 827-833.

[28] Michal R Wrobel. 2013. Emotions in the software development process. In HSI. IEEE, 518-523. 